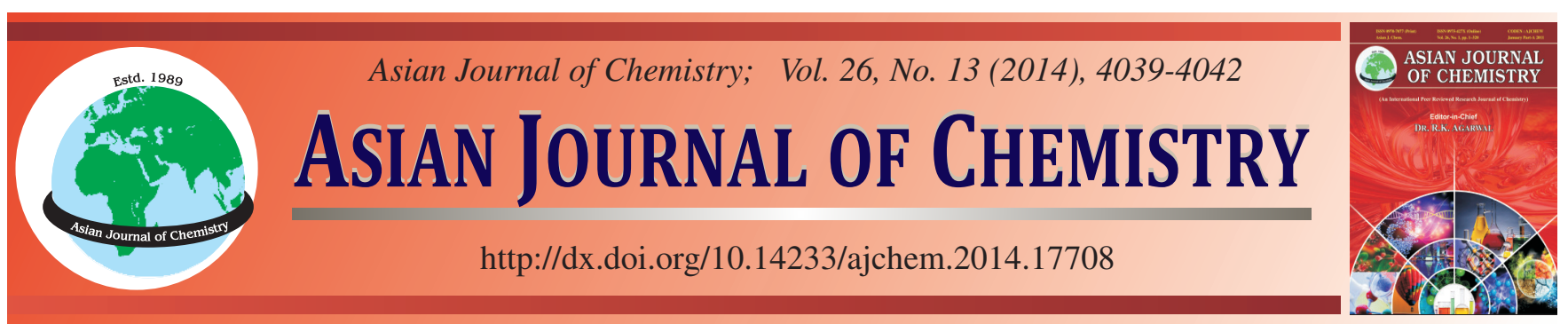

\title{
Coordinate System Alignment Algorithm of Advanced Remote Handling System for Decommissioning of Nuclear Facility $\dagger$
}

\author{
Donguun Hyun ${ }^{*}$, Geun-Ho Kim, Jong-Hwan Lee, Kwan-Seong Jeong, Byung-Seon Choi and Jeikwon Moon
}

Decontamination \& Decommissioning Research Division, Korea Atomic Energy Research Institute, 989-111 Daedeok-daero, Yuseong-gu, Daejeon 305-353, Republic of Korea

*Corresponding author: Fax: +82 42 8688062; Tel: +82 42 8688081; E-mail: dongjunn@kaeri.re.kr

\begin{abstract}
A novel coordinate system alignment algorithm is proposed for an advanced remote handling system which is the key solution to dismantle heavy, chemically contaminated and highly radioactive components during nuclear facility decommissioning. A high radiation field and chemical contamination extremely limit hands-on operations by workers for safety and radiation protection and a heavy component requires high payload capacity and long lead-time for dismantling operations, which are not suitable for human workers. Over the last decades, several remote handling systems have been developed to dismantle highly activated heavy components in nuclear facility decommissioning and have achieved specific goals. However, the well-known disadvantages of the remote operation, which are a limited cognition and the complex operation, still obstructs the expanding application of a remote handling system. The algorithm in this paper presents an original methodology using the polar scan matching algorithm and the multi-rate extended Kalman filtering algorithm to align coordinate systems of the known 3D digital model and 3D scanning data over the remote workshop in a remote handling system composed of systems having different operational periods and can largely contribute to an advanced remote handling system with a user friendly interface.
\end{abstract}

Keywords: Coordinate system alignment, Remote handling system, Decommissioning, Radioactive and chemical waste treatment.

\section{INTRODUCTION}

Many of nuclear power plants under operation were constructed several decades ago when the nuclear industry experienced the most activated period and have almost reached their license termination. Therefore, nuclear facility decommissioning has become an emerging issue to the contrary of the activated period. In the context of the nuclear industry, only a few advanced countries have experiences in nuclear facility decommissioning and have accumulated related technologies ${ }^{1}$. However, to deal with the decommissioning of upcoming numerous nuclear facilities effectively, various related technologies still have to be developed and improved. Remote handling technologies as a branch of decommissioning technologies is absolutely necessary to dismantle heavy, chemically contaminated and highly radioactive components in a nuclear power plant because radiation and chemical hazards protection for workers limits access to the high radiation field and the heavy components require a high payload capacity and long leadtime for dismantling operations.
A multi-degree-of-freedom manipulator takes an important part in remote handling technologies and is applied to the dismantling of complex and highly radioactive structures. The remotely controlled manipulator requires the highest level of technology out of remote handling technologies because the manipulator has to realize complex motions according to the intention of the remote operator and the remote operator has to cognize clearly depending on limited images provided by monitoring cameras and has to control the motion of the manipulator precisely with insufficient information over the remote workshop. Previously developed manipulators have successfully achieved most of their goals in the context of the hardware, which are payload capacity, workspace and versatility. However, the manipulators still have a considerable problem in their user interface and thus the remote operator needs to spend a long time training to satisfy the required level of skill and only a few persons out of many trainees can be adopted as a remote operator. The remote operator may experience significant mental fatigue, make frequent mistakes and take a long time to complete an operation. 
A user friendly interface with the dismantling manipulator focuses on how to reduce the mental load of the remote operator. The user interface methodology combining augmented reality and haption was studied by Dejong to reduce mental transformation $^{2}$ and similar approaches have been studied ${ }^{3-6}$. Previously referred researches require a common technology to transform a real workshop into a virtual 3D model based on 3D scanning data.

The algorithm in this paper presents a methodology to align the coordinate system of a known virtual 3D model with the coordinate system of 3D scanning data over a real workshop in real-time when the dismantling system consists of several systems having different operational periods. In the following experimental section, an overview of the advanced remote handling system and basic algorithms are introduced. In the results and discussion section, the resulting algorithm presented in this paper is described.

\section{EXPERIMENTAL}

Overview of advanced remote handling system: An advanced remote handling system is similar to a conventional remote manipulator system with mechanical specifications adequate to prearranged dismantling operations and equipped with a $3 \mathrm{D}$ scanning system at the arm of the manipulator (Fig. 1). The 3D scanning system consists of a 2D laser scanner and a tilting mechanism and thus the system can obtain 3D data by combining $2 \mathrm{D}$ scanning data at each tilted plane, which takes place at the arm of the manipulator so that the system can observe the end effecter and the operational target at the best position.

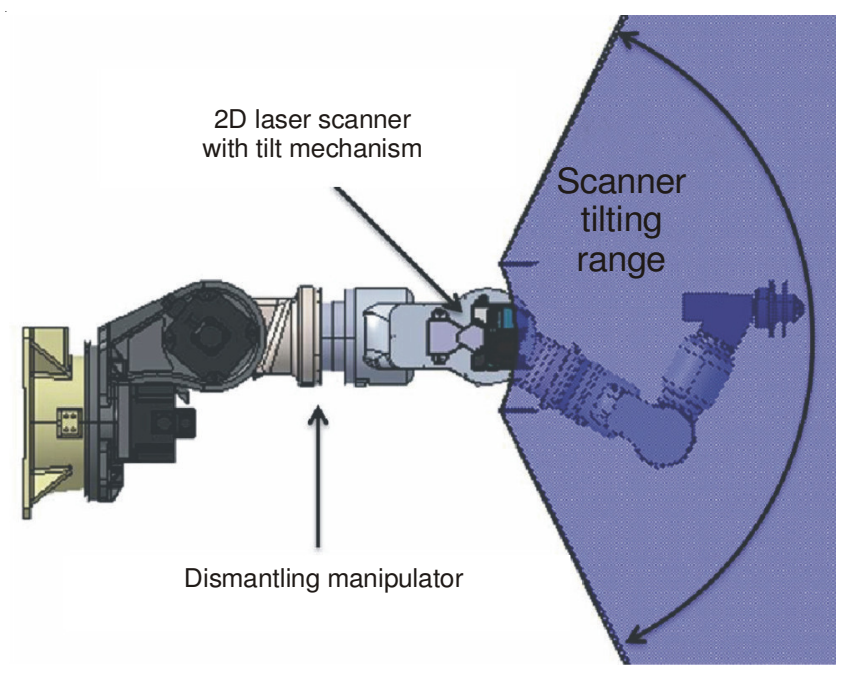

Fig. 1. Manipulator with 3D scanning system

Polar scan matching algorithm: The scan matching algorithm is required to align the coordinate systems of a known 3D digital model and 3D scanning data over the remote workshop. The Iterated Closest Point (ICP) has been widely used as the scan matching algorithm, but takes too much time for processing ${ }^{7}$. The polar scan matching algorithm was presented by Albert Doisi and Lindsay Kleeman for faster processing ${ }^{8}$. The coordinate system alignment algorithm in this paper adopts the polar scan matching algorithm, the processing steps of which are as follows: 1) Obtain scan data from the 3D scanning system, 2) Estimate scan data from the known 3D digital model and estimated manipulator pose, 3) Translational estimation with linearized least squares, 4) Rotational estimation with window search, 5) Iterate $\mathrm{N}$ times.

Multi-rate extended Kalman filter algorithm: An advanced remote handling system requires a multi-rate sampling method to deal with asynchronous systems because the scan matching system is expected to spend about 1 to $10 \mathrm{~s}$ for $3 \mathrm{D}$ scanning data acquisition and a unit computing process and the motion control system of the dismantling manipulator is expected to spend about 0.001 to $0.01 \mathrm{~s}$ to process a unit control loop. The multi-rate extended Kalman filter algorithm had been proposed by Armesto et al. ${ }^{9}$ and has been adopted by the tracking algorithm for 3-D pipeline mapping ${ }^{10}$. The algorithm is most suitable for an advanced remote handling system. The processing steps of the multi-rate extended Kalman filter algorithm are as follows: 1) Initialize empty measurement vector $\mathrm{z}_{\mathrm{k}}$ and matrix $\mathrm{H}_{\mathrm{k}}, 2$ ) If $\mathrm{z}_{1}$ measurement value exists, add $\mathrm{z}_{1}$ to measurement vector $z_{k}$, add $H_{1}$ to $H_{k}$ and add $r_{1}$ to $r_{k}$, 3) If $z_{2}$ measurement value exists, add $z_{2}$ to measurement vector $z_{k}$ add $\mathrm{H}_{2}$ to $\mathrm{H}_{\mathrm{k}}$ and add $\mathrm{r}_{2}$ to $\mathrm{r}_{\mathrm{k}}$, 3) Iterate as many times as the size of the measurement vector, 4) Predict the current state vector $x_{k}$ and covariance matrix $\left.P_{k}, 5\right)$ Compute extended Kalman filtering matrix $\mathrm{K}_{\mathrm{k}}$ with $\mathrm{H}_{\mathrm{k}}, \mathrm{P}_{\mathrm{k}}, \mathrm{R}_{\mathrm{k}}, 6$ ) Estimate the current state vector $x_{k}$ and covariance matrix $P_{k}$.

\section{RESULTS AND DISCUSSION}

Coordinate system alignment algorithm: The coordinate system algorithm in this paper aligns the coordinate systems of the known 3D digital model and 3D scanning data over the remote workshop and synchronizes the aligned coordinate system with the coordinate system of the dismantling manipulator which varies even more rapidly than the aligned coordinate system according to the motion of the manipulator. The algorithm consists of the coordinate system alignment loop and the manipulator control loop as shown on Fig. 2. The update rate of the two loops is different and thus the multirate extended Kalman filter combines the two loops with the multi-rate sampling methodology.

Fig. 3 shows an example application of an advanced remote handling system which is to cut the head of the reactor pressure vessel (RPV). To cut the RPV head precisely with the manipulator, accurate relative coordinates between the end effecter and the RPV head are required and can be found through simple multiplication of transformation matrices representing relative coordinates from the end effecter to the RPV head. The transformation matrices can be easily found by measuring the position of the equipment at their installation and computing rapidly varying positions based on the joint angles of the equipment measured with sensors. However, a coordinate system alignment algorithm is still required to obtain accurate relative coordinates because of measurement errors and uncertainty.

Fig. 4 shows the aligning process using reference geometry and 3D scan data by comparing the two data points in the polar coordinate system with linearized least squares. The left graph represents the $\mathrm{x}-\mathrm{y}$ plane with millimeter unit 


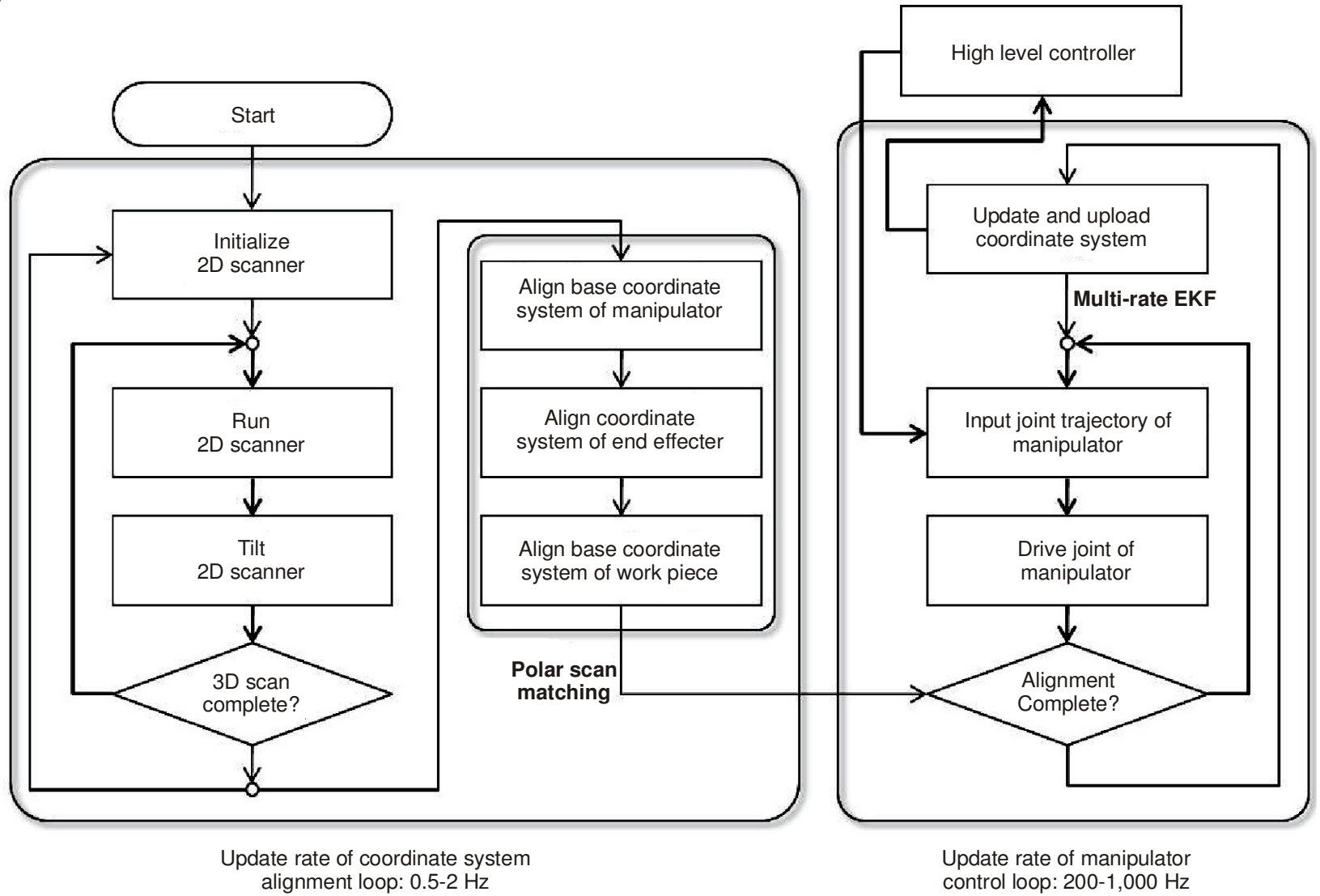

Fig. 2. Coordinate system alignment algorithm

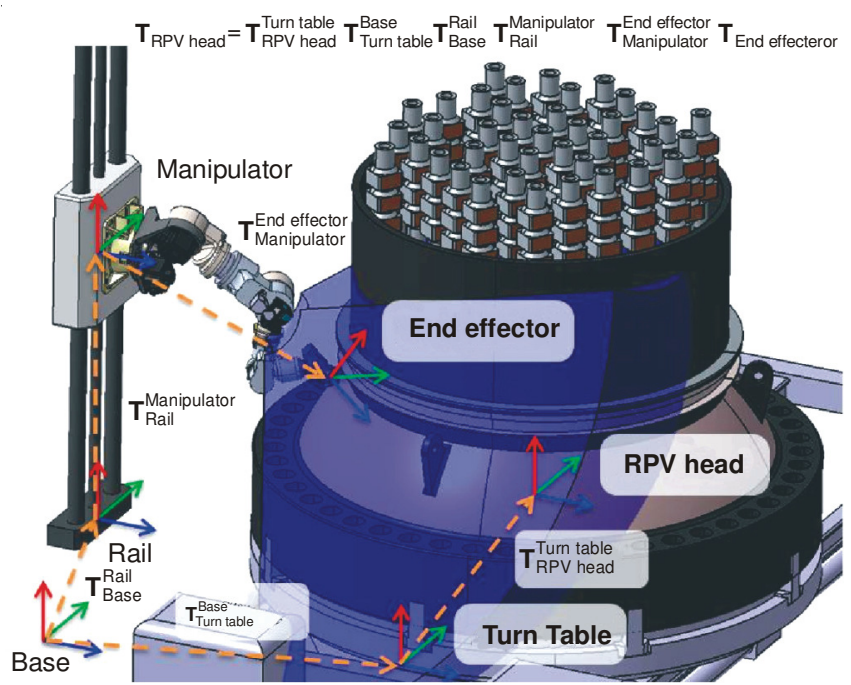

Fig. 3. Application of advanced remote handling system

and the right graph represents the -r plane with radian and millimeter unit. At the initial step, the 3D scan data are far from the reference geometry both position and orientation. The aligning process transfers the $3 \mathrm{D}$ scan data and the reference geometry from Cartesian to the polar coordinates and repeats translational estimation and rotational estimation predefined $\mathrm{N}$ times. At the final step, the alignment result matches the reference geometry well.
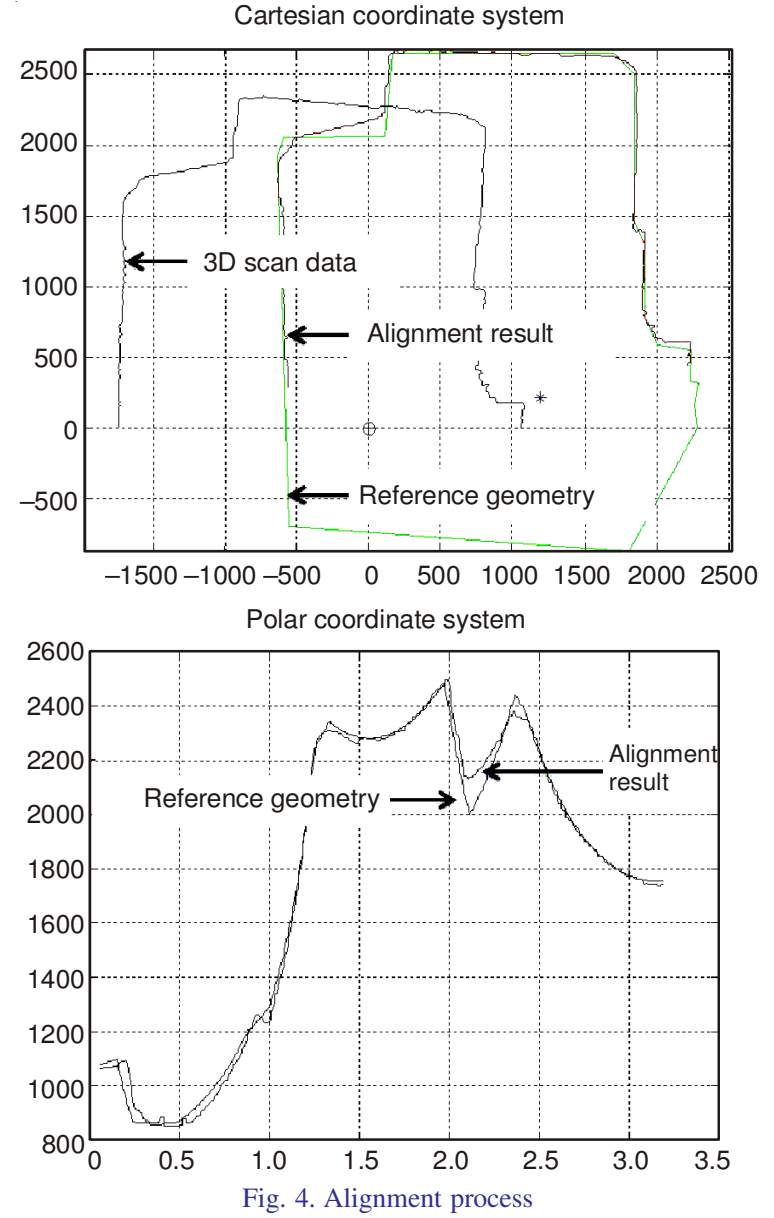


\section{Conclusion}

The proposed coordinate system alignment algorithm successfully aligns relative coordinates between the end effecter of an advanced remote handling system and the cutting object despite asynchronous constituent systems and an uncertain reference geometry. This paper can contribute to a user friendly interface of an advanced remote handling system to dismantle heavy, chemically contaminated and highly radioactive components of a nuclear facility.

\section{ACKNOWLEDGEMENTS}

The research was supported by the Nuclear R\&D Program through the Ministry of Science, ICT \& Future Planning.

\section{REFERENCES}

1. Radioactive Waste Management Committee, Remote Handling Techniques in Decommissioning (2011).

2. P. Brian, Int. J., 33, 187 (2006).

3. J. Pretlove, Int. J., 25, 401 (1998).

4. P.J. Besl and N.D. McKay, IEEE Trans. Pattern Anal. Mach. Intell., 14, 239 (1992).

5. T. Yamamoto, N. Abolhassani, S. Jung, A.M. Okamura and T.N. Judkins, Int. J. Med. Robot., 8, 45 (2012).

6. D.W.F. van Krevelen and R. Poelman, Int. J. Virtual Reality, 9, 1 (2010).

7. S. Corazza, L. Mundermann, E. Gambaretto, G. Ferrigno and T.P. Andriacchi, Int. J. Comput. Vis., 87, 156 (2010).

8. A. Diosi and L. Kleeman, Int. J. Robot. Res., 26, 1125 (2007)

9. L. Armesto, J. Tornero and M. Vincze, Int. J. Robot. Res., 26, 577 (2007).

10. D. Hyun, H.S. Yang, H.-S. Park and H.-J. Kim, Mechatronics, 20, 213 (2010). 